\title{
Assessment of human fetal heart hemodynamics during prenatal development
}

\author{
Huseyin C Yalcin ${ }^{1}$, Huseyin Enes Salman², Reema Yousef Kamal ${ }^{3}$, \\ ${ }_{1}^{1}$ Biomedical Research Center, Qatar University, PO Box 2713, Doha, QATAR \\ ${ }^{1}$ Department of Mechanical Engineering, TOBB University of Economics and Technology, Ankara, TURKEY \\ ${ }^{2}$ Pediatric Cardiology Division, Hamad General Hospital, Hamad Medical Corporation, Doha, QATAR
}

\begin{abstract}
BACKGROUND
\end{abstract}

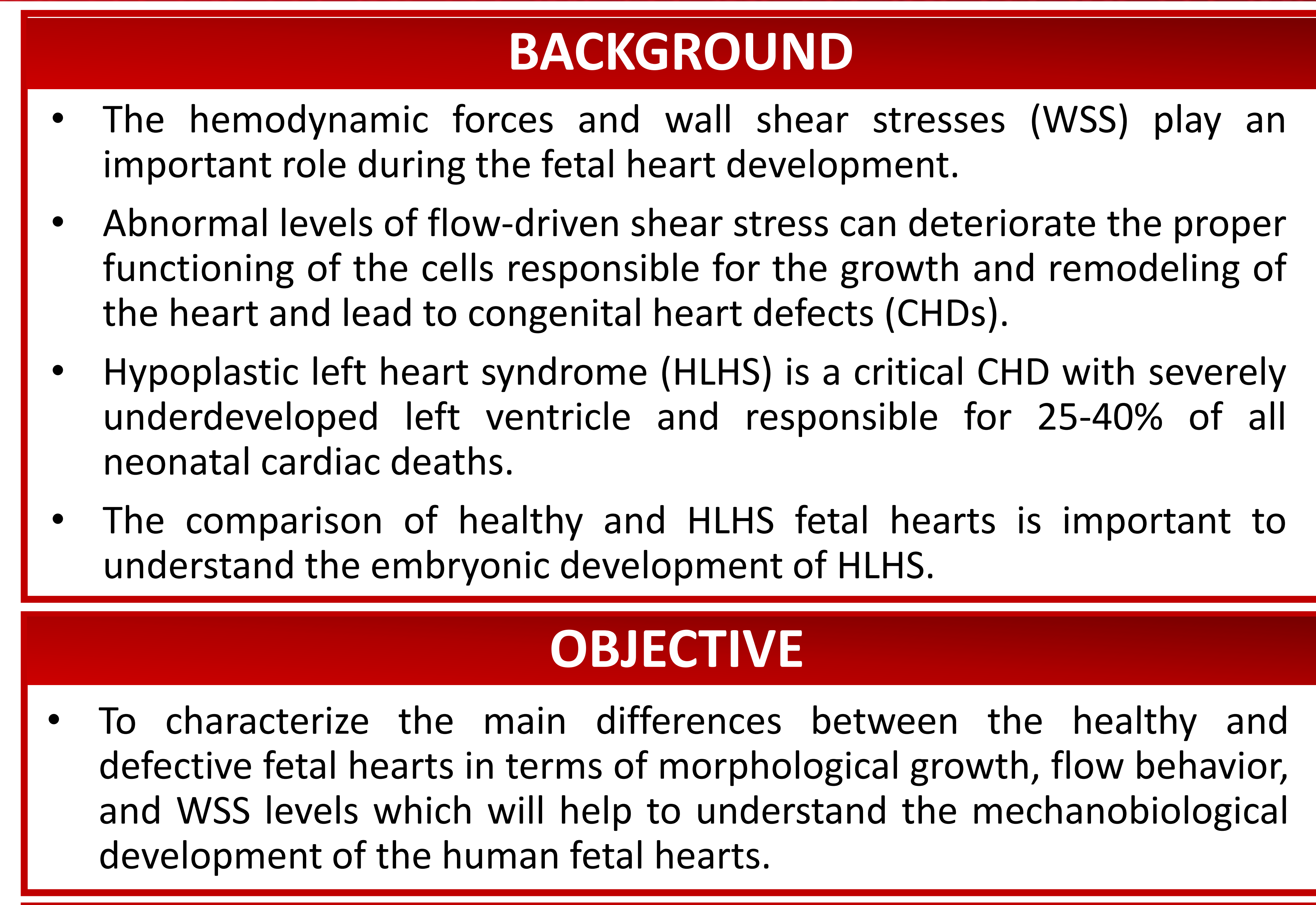

\section{METHODS}

- Generation of model geometry and defining boundary conditions:

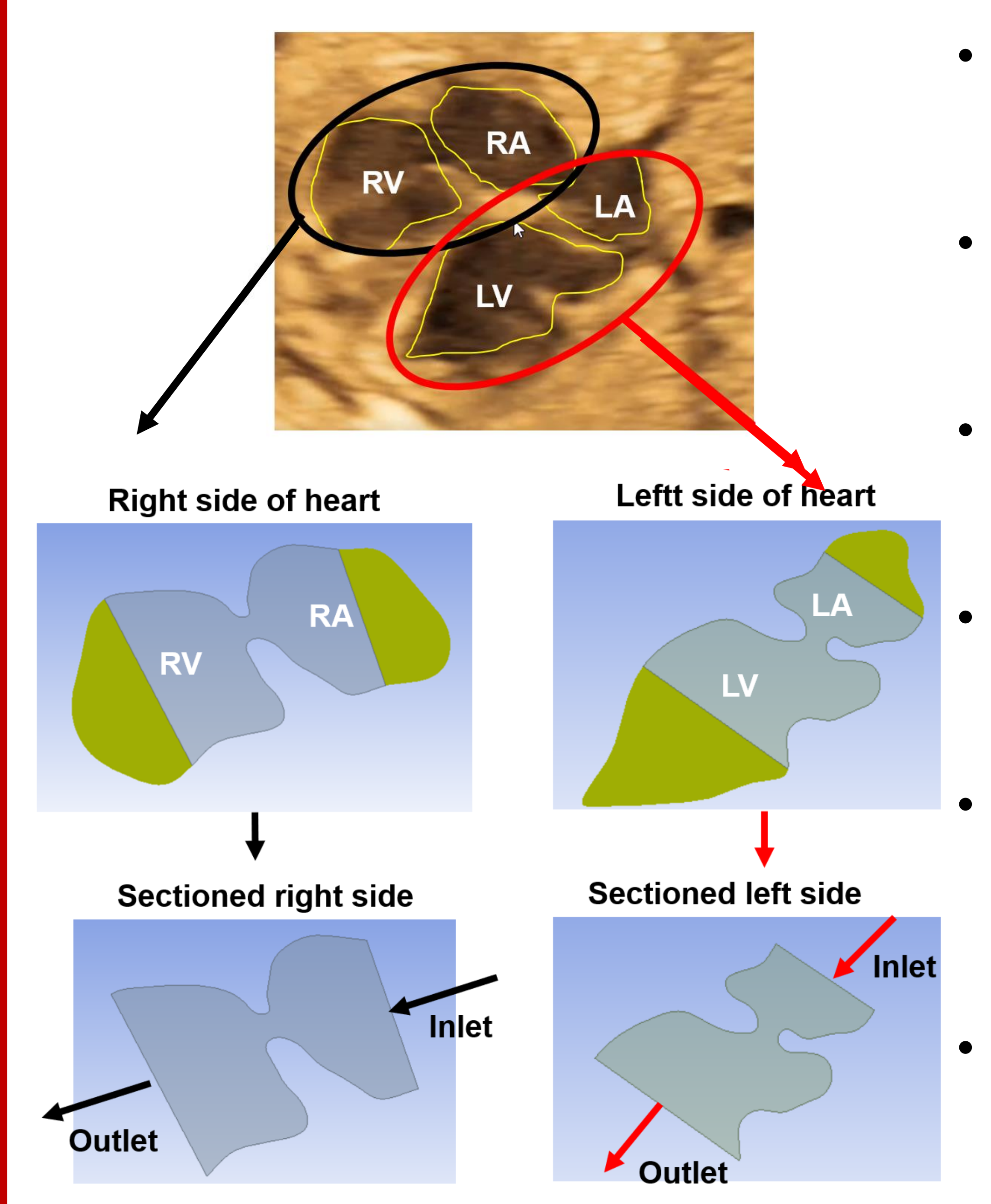

\begin{abstract}
Medical images are used to determine the borders of the fetal heart chambers.

- Left and right sides of healthy and defective hearts are investigated separately.

About half of the chambers is removed to apply the inlet and outlet boundary conditions.

Inlet flow conditions are determined using Doppler ultrasound velocity measurements.

Computational fluid dynamics (CFD) simulations are performed using ANSYS Fluent solver for obtaining the flow field inside the heart.

Flow velocities, pressures, WSS levels, and heart chamber areas are compared for the healthy and HLHS hearts.
\end{abstract}

- Meshing the generated model geometries for the CFD simulations:

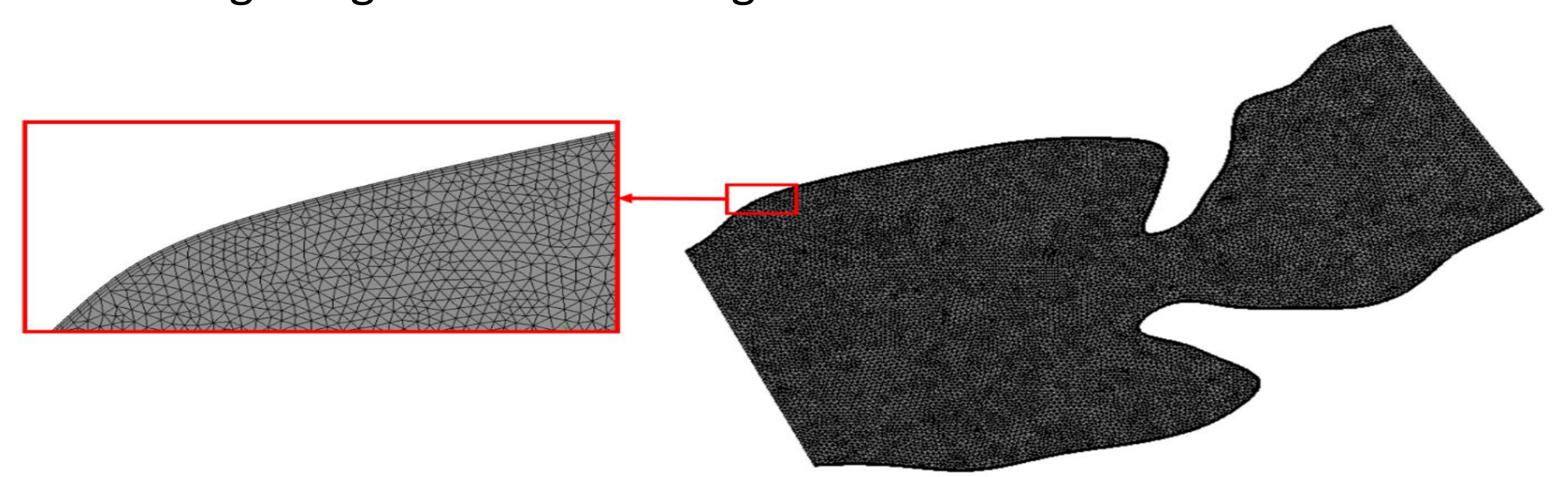

A sample mesh used to solve the governing flow equations in the CFD analysis. The mesh density is increased near the walls in order to improve the solution accuracy.
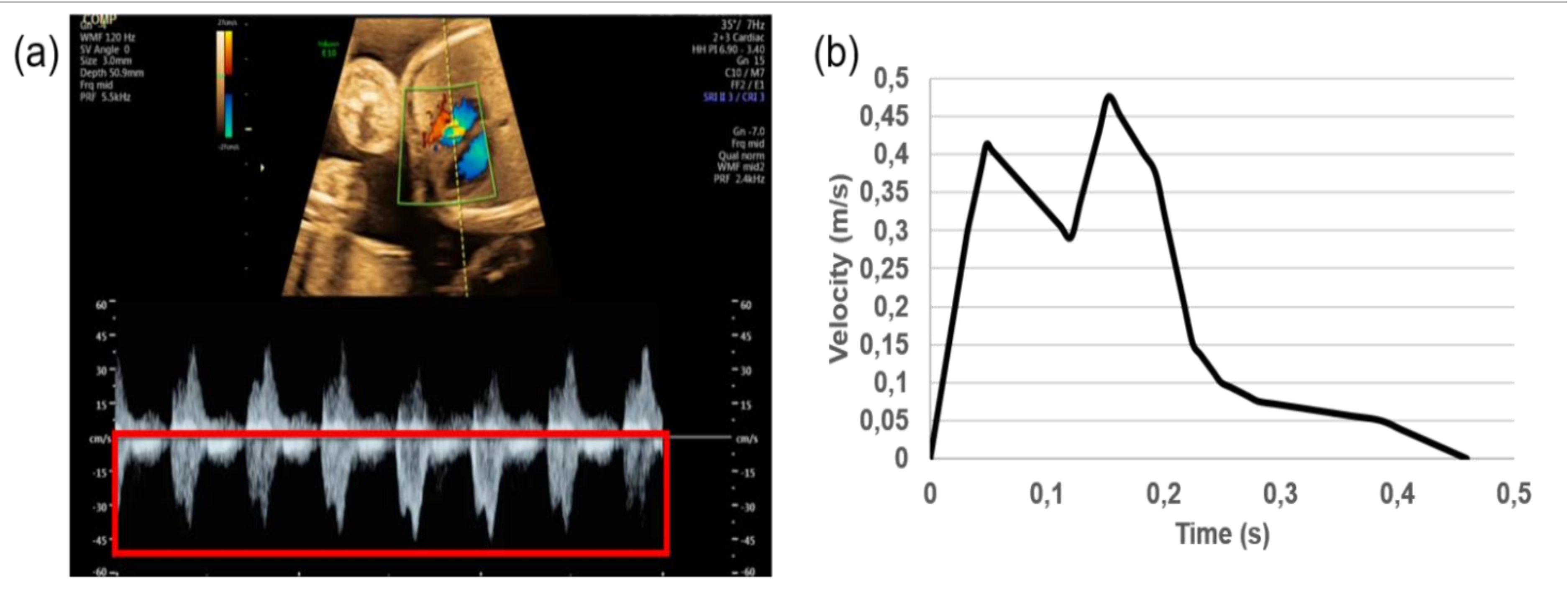

A sample Doppler ultrasound measurement in a human fetal heart.

(a) The red box shows the flow waveforms measured in the mitral valve. (b) Mitral valve flow waveform as a function of time for one cardiac cycle.

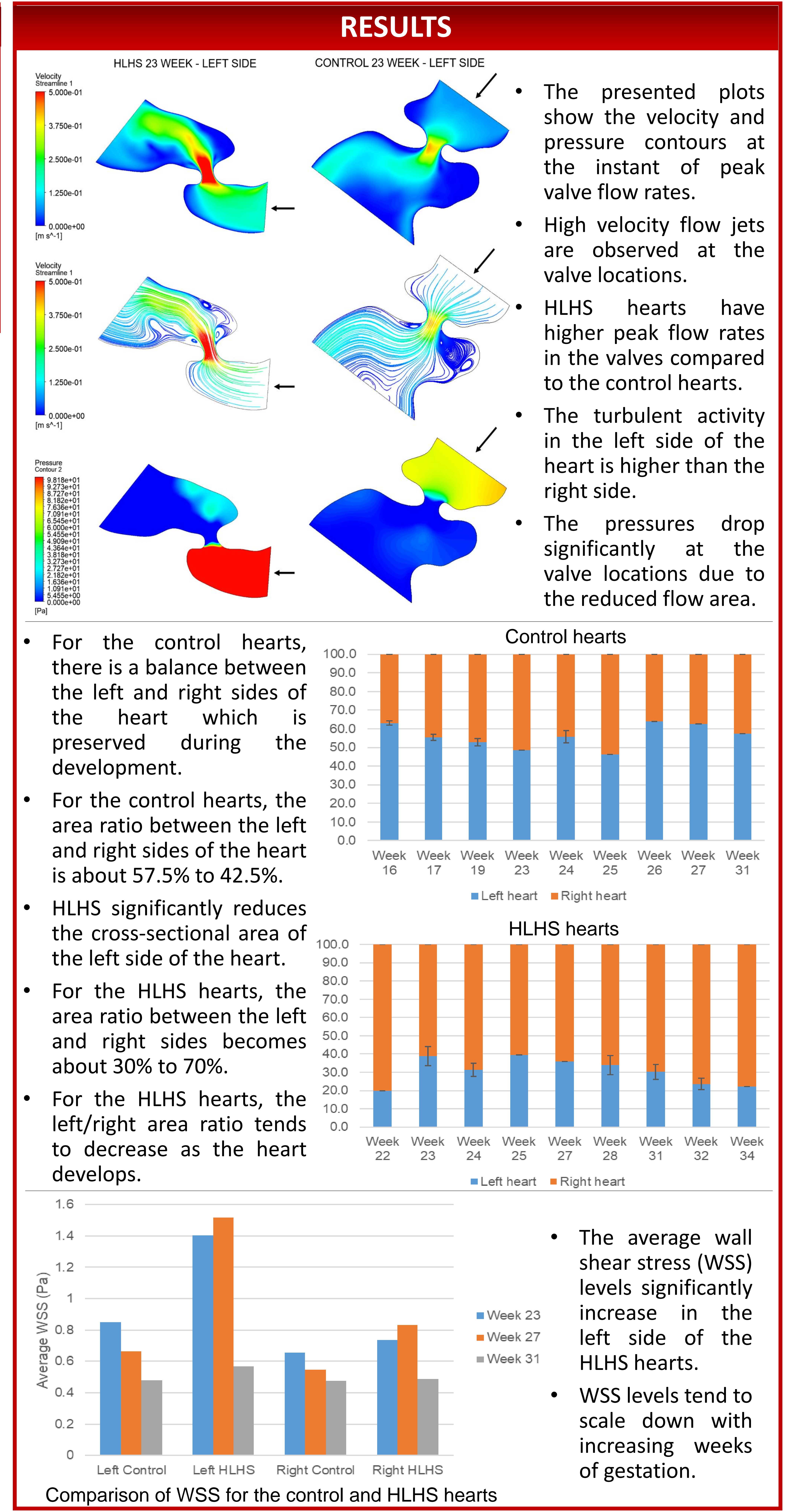

CONCLUSION

- The presence of CHDs alters the biomechanical environment and hemodynamics in the fetal hearts.

- HLHS leads to a significant increase in the WSS levels, particularly in the left side of the fetal hearts.

- In HLHS hearts, the ratio of cross-sectional area between the left and right sides of the heart changes drastically due to the underdeveloped left side. 